\title{
Le regard des mères sur les attitudes, les compétences et les connaissances sur le TSA du personnel scolaire qui influencent l'intégration en classe ordinaire de leur enfant présentant un TSA
}

\section{The outlook of mothers on the attitudes, knowledge of TSA and skills of primary school staff influencing integration into ordinary classes of their child with TSA}

\section{Magalie Boucher-Gagnon et Catherine des Rivières}

Volume 44, numéro 2, 2015

URI : https://id.erudit.org/iderudit/1039254ar

DOI : https://doi.org/10.7202/1039254ar

Aller au sommaire du numéro

Éditeur(s)

Revue de Psychoéducation

ISSN

1713-1782 (imprimé)

2371-6053 (numérique)

Découvrir la revue

Citer cet article

Boucher-Gagnon, M. \& des Rivières, C. (2015). Le regard des mères sur les attitudes, les compétences et les connaissances sur le TSA du personnel scolaire qui influencent l'intégration en classe ordinaire de leur enfant présentant un TSA. Revue de psychoéducation, 44(2), 219-243.

https://doi.org/10.7202/1039254ar
Résumé de l'article

À ce jour au Québec, aucune étude n'a porté sur l'expérience des parents concernant l'intégration scolaire en classe ordinaire de leur enfant ayant un TSA. Il s'agit d'une limite importante des connaissances, surtout dans le contexte où le gouvernement souhaite être ouvert à l'expertise des parents. L'objectif de cette étude est donc d'analyser plus en profondeur, à partir des propos de 18 mères, ce qui, selon elles, a influencé le déroulement de l'intégration scolaire de leur enfant présentant un TSA. Les résultats obtenus montrent que les attitudes, les compétences et les connaissances à propos du TSA de la part du personnel scolaire sont déterminantes, selon les mères, dans l'intégration de leur enfant. Les conclusions suggèrent également que, toujours selon les mères, le personnel scolaire a de la difficulté à s’adapter à leur nouvelle réalité d'intégrer des élèves avec des besoins particuliers.
Tous droits réservés $@$ La Corporation de la Revue Canadienne de Psycho-Éducation, 2015
Ce document est protégé par la loi sur le droit d'auteur. L'utilisation des services d'Érudit (y compris la reproduction) est assujettie à sa politique d'utilisation que vous pouvez consulter en ligne. 


\title{
Le regard des mères sur les attitudes, les compétences et les connaissances sur le TSA du personnel scolaire qui influencent l'intégration en classe ordinaire de leur enfant présentant un TSA
}

\author{
The outlook of mothers on the attitudes, knowledge of TSA \\ and skills of primary school staff influencing integration into \\ ordinary classes of their child with TSA
}

M. Boucher-

Gagnon ${ }^{1}$

C. des Rivières²

1 Département de psychologie, Université du Québec à Montréal

2 Département de sociologie, Université du Québec à Montréal

\section{Correspondance :}

Catherine des Rivières,

Département de sociologie, Université du Québec à Montréal, C.P. 8888 , succ. Centre-Ville, Montréal (Québec) H3C 3P8 desrivieres.catherine@uqam.ca

Tél. : 514 987-3000, poste 2534 Téléc. : 514 987-4638

\section{Résumé}

À ce jour au Québec, aucune étude n'a porté sur l'expérience des parents concernant l'intégration scolaire en classe ordinaire de leur enfant ayant un TSA. II s'agit d'une limite importante des connaissances, surtout dans le contexte où le gouvernement souhaite être ouvert à l'expertise des parents. L'objectif de cette étude est donc d'analyser plus en profondeur, à partir des propos de 18 mères, ce qui, selon elles, a influencé le déroulement de l'intégration scolaire de leur enfant présentant un TSA. Les résultats obtenus montrent que les attitudes, les compétences et les connaissances à propos du TSA de la part du personnel scolaire sont déterminantes, selon les mères, dans l'intégration de leur enfant. Les conclusions suggèrent également que, toujours selon les mères, le personnel scolaire a de la difficulté à s'adapter à leur nouvelle réalité d'intégrer des élèves avec des besoins particuliers.

Mots-clés : Trouble du Spectre de l'Autisme (TSA), intégration scolaire, perception des mères, attitude du personnel scolaire et connaissance du personnel scolaire.

\section{Abstract}

The parents are important actors in the educational success or their child, simply because they are the person who knows them best. With that in mind, there is no study, in Quebec, which leads us to think that parents can be of any help with the school integration of their child affected by ASD. This is an important limit considering that the government pays a lot of expertise to parents. The goal of the study is to analyse more deeply the experience of 18 mothers, this, they say, has influenced the course of the school integration of their child with ASD. The obtained results show that the attitudes, skills and knowledge about TSA are a major role in mothers perception with regard to the integration of their child. Unfortunately, the findings 


\section{0}

also state mothers think that the school staff seem to have difficulty to adapt to their new reality, which is to integrate students with special needs.

Keywords: Autism Spectrum Disorder (ASD), mainstream school, maternal percep-
tion, attitude of school staff and school staff knowledge.

\section{Introduction}

Depuis les dernières décennies, la conception de l'éducation des personnes avec des besoins particuliers a beaucoup évolué passant de l'institutionnalisation et la marginalisation au désir de les intégrer dans la société comme des personnes à part entière. À cet effet, en 1999, le gouvernement québécois a modifié la politique de l'adaptation scolaire pour permettre à tous les élèves avec des besoins particuliers de bénéficier d'une éducation égalitaire et de qualité (MEQ, 1999). Plus récemment, les politiques gouvernementales sur l'éducation mettent l'accent sur les pratiques d'intégration dites inclusives. Ainsi, peu importe les besoins de l'enfant, ce dernier devrait pouvoir être intégré dans une classe ordinaire (MELS, 2008). Ce changement de perspective, associé à la hausse d'environ $20 \%$ de la prévalence des élèves handicapés ou en difficultés d'adaptation ou d'apprentissage (EHDAA) dans le milieu scolaire entre les années 2002-2003 et 2009-2010 constituent sans aucun doute des éléments centraux dans la transformation du " portrait » de la classe ordinaire, celle-ci se composant de plus en plus fréquemment d'enfants présentant des défis particuliers (MELS, 2010).

Parmi les éléments qui expliquent la hausse globale des élèves HDAA, on note la hausse du nombre d'élèves présentant un trouble du spectre de l'autisme (TSA) 1 qui est désormais le handicap le plus recensé en milieu scolaire. Au Québec, depuis 2009-2010, l'augmentation de la prévalence est de $26 \%$. Il est donc fréquent de rencontrer des élèves ayant un TSA dans une classe ordinaire, car ceux-ci sont intégrés dans une proportion de $41 \%$ (Noiseux, 2012). Dans le cadre d'une pratique dite inclusive, les personnes impliquées auprès de ces enfants doivent s'adapter à leur réalité et ajuster leur pratique professionnelle aux spécificités de chacun d'entre eux. Plusieurs études portant sur l'intégration scolaire des enfants ayant un TSA montrent en effet que lorsque le personnel scolaire manque de connaissance sur le TSA, cela entraîne l'augmentation de croyances erronées (Helps, NewsomDavis et Callias, 1999; MacFarlane et Woolsfon, 2013; McGregor et Campbell, 2001) et un manque de sensibilité envers les particularités de l'enfant (Emam et Farrell, 2009; Whitaker, 2007). Ces études ont été majoritairement effectuées auprès d'enseignantes ${ }^{2}$, de professionnelles du milieu scolaire ou de la direction.

1. Le terme trouble du spectre de l'autisme (TSA) est utilisé dans ce texte pour respecter les nouvelles conventions de catégorie diagnostique prescrite dans la récente version du DSM- 5 de l'American Psychiatric Association (APA, 2013). L'acronyme TSA sera également privilégié afin d'alléger la lecture de l'article.

2. Le féminin sera employé afin de représenter la majorité des enseignantes, des accompagnatrices et des directrices qui travaillent dans le milieu scolaire. 
À notre connaissance, aucune étude n'a porté sur la perception qu'ont de l'intégration scolaire, en classe ordinaire, les parents québécois d'enfant ayant un TSA. Pourtant, selon le gouvernement du Québec, le regard que posent les parents sur l'intégration devrait constituer un élément clé de la compréhension de cette problématique, car ceux-ci sont les principaux acteurs de la réussite de leur enfant et sont les mieux informés des besoins de ce dernier (MELS, 2010; MEQ, 2003; MSSS, 1996).

Notons également que, bien que les échantillons des quelques études qui ont analysé l'expérience de parents d'enfant ayant un TSA intégré en milieu ordinaire étaient majoritairement constitués de mères, une seule recherche a porté exclusivement sur l'expérience de celles-ci (Little, 2003). II s'agit à notre avis d'une limite importante des connaissances, car les mères ont une expérience susceptible d'être fort différente de celle des pères puisqu'elles interagissent différemment de ces derniers avec le personnel intervenant auprès de leur enfant (Baker-Ericzèn, Brookman-Frazee et Stahmer, 2005; Granger, 2011; Sabourin, 2012). Cette recherche vise donc à documenter les perceptions des mères en analysant de façon plus spécifique les attitudes, les compétences et les connaissances sur le TSA du personnel scolaire qui selon elles, ont influencé le déroulement de l'intégration scolaire en classe ordinaire de leur enfant présentant un TSA. Cette démarche permettra de mettre en lumière les sources de satisfactions et d'insatisfactions des mères.

\section{Trouble du Spectre de l'Autisme (TSA)}

Selon les nouveaux critères définis dans la 5è édition du manuel DSM (APA, 2013), le TSA se caractérise par une altération significative et persistante de la communication et des interactions sociales associées à des patrons de comportements, d'activités ou d'intérêts caractérisés par leur nature restreinte, stéréotypée ou répétitive. Les symptômes doivent se manifester tôt dans l'enfance, mais certains peuvent se déclarer seulement lorsque les exigences sociales dépassent les compétences de l'enfant.

L'une des particularités du TSA est la grande diversité des profils que ce trouble regroupe (Schwartz, Sandall, McBride et Boulware, 2004; Simpson, BoerOtt et Smith-Myles, 2003). De plus, les enfants présentant un TSA ont souvent des difficultés associées telles que des problèmes de sommeil, des particularités alimentaires, des problèmes moteurs et certains troubles psychiatriques (Rogé, 2008). Il est clair que ces disparités dans les profils constituent un défi supplémentaire pour les parents et le personnel scolaire lorsque vient le temps de cibler les besoins de ces enfants.

\section{Intégration scolaire : perception des parents d'enfant ayant un TSA}

Les études ayant mesuré la satisfaction des parents d'enfant ayant un TSA concernant l'intégration scolaire démontrent que ces derniers sont souvent insatisfaits des services scolaires reçus (Brewin, Renwick et Schormans, 2008; Starr et Foy, 2012; Starr, Foy, Cramer et Singh, 2006; Whitaker, 2007). L'une des sources de cette insatisfaction est l'attitude du personnel scolaire qui adopte, 
d'après les parents, une posture d'expert et une logique administrative qui limite les échanges et qui dénote parfois un manque de respect envers les parents (Stoner et al., 2005). Plusieurs parents ont d'ailleurs l'impression de devoir adopter une posture « revendicatrice » afin que le personnel réponde aux besoins de leur enfant (Stoner, Angell, House et Bock, 2007). L'étude de Renty et Roeyers (2006), qui a porté sur la satisfaction des parents à l'égard des services scolaires, montre que les parents qui perçoivent leur expérience comme étant difficile ont généralement eu du mal à accéder aux services requis pour leur enfant (par ex. évaluation, intervention, accès à des professionnels, etc.), ont fait face à un manque d'information sur le TSA et à un manque de soutien en contexte d'intégration scolaire. Toujours selon ces auteurs, les parents accordent une grande importance à leur implication dans le processus scolaire, mais souhaitent que celle-ci se fasse dans un esprit de collaboration avec l'équipe-école. Ils soulignent également qu'une mise en place adéquate des services nécessite, de la part du personnel, une connaissance des besoins de l'enfant, ce qui requiert à son tour une connaissance minimale des particularités du TSA. Selon Cappe (2012), la mise en place de services adaptés permet aux parents de développer et de maintenir des relations harmonieuses avec le personnel. Une autre recherche met également en évidence que les parents ont besoin de continuité et de soutien lors du processus d'intégration scolaire de leur enfant (Sapin, Thommen et Wiesendanger, 2007).

Au Québec, Rousseau, Dionne, Vézina et Drouin (2009) ont mené une étude sur l'intégration scolaire mesurant la satisfaction des parents d'enfant présentant des besoins particuliers, dont faisaient partie entre autres des parents d'enfant ayant un TSA. Les résultats de cette recherche, qui étaient similaires à ceux de recherches menées auprès de personnes ayant un TSA en Belgique (Renty et Roeyers, 2006), au Canada (Brewin, Renwick et Schormans, 2008; Siklos et Kern, 2006; Starr et Foy, 2012; Starr, Foy, Cramer et Singh, 2006) et aux États-Unis (Stoner, Angell, House et Bock, 2007; Stoner et al., 2005) ont rapporté l'importance que les parents accordent au fait d'avoir une bonne collaboration avec l'école, à la capacité d'adaptation dont fait preuve le milieu scolaire et au fait de développer des relations positives avec le personnel. Ces études mettent en évidence que les compétences professionnelles et l'attitude des personnes intervenant auprès de l'enfant ayant un TSA ont une influence sur la satisfaction des parents de ce dernier. II apparaît donc pertinent de se pencher sur les caractéristiques du personnel scolaire québécois qui ont, selon les mères, influencé le déroulement de l'intégration de leur enfant présentant un TSA.

\section{Le personnel scolaire}

Les recherches actuelles sur l'intégration scolaire se centrent majoritairement sur la perception des différents membres du personnel scolaire, soit la direction, les enseignantes et les accompagnatrices ${ }^{3}$ concernant les facteurs

3. Le terme " accompagnatrice » est utilisé afin de simplifier la compréhension des lecteurs, car dans la littérature actuelle le terme accompagnatrice, assistant-enseignant, coordonnateur, technicien en éducation spécialisé (TES) et éducateur sont utilisés pour évoquer pour le même rôle dans le contexte d'intégration soit accompagner l'enfant en classe. 
qui facilitent l'intégration. La section qui suit présente les principaux résultats de ces recherches. Bien que celles-ci ne tiennent généralement pas compte du regard que posent les parents sur cette intégration, elles nous renseignent sur le rôle que chacun des acteurs peut jouer dans le déroulement de l'intégration de l'enfant.

\section{La direction d'école}

Plusieurs études ont confirmé que la direction d'école joue un rôle crucial dans l'intégration des enfants ayant un TSA en raison de son pouvoir décisionnel qui permet de mettre en place des mesures susceptibles d'aider les enfants intégrés et le personnel dont la tâche est d'aider ces enfants. En effet, des études mettent de l'avant qu'une direction possédant de bonnes connaissances et une bonne compréhension du TSA comprend mieux les besoins de ces enfants et tend donc davantage à fournir les services et les ressources adéquates pour le personnel scolaire et l'enfant (Horrocks, White et Roberts, 2008; Symes et Humphrey, 2011). Par exemple, la formation offerte au personnel scolaire (Eldar, Talmor et WolfZukerman, 2010; lalongo, 2007; Symes et Humprey, 2011) et la possibilité d'avoir un temps de préparation et de planification nécessaire pour adapter l'environnement ou l'enseignement (Ruel, 2014) sont des mesures pouvant être offertes par la direction et qui semblent avoir une influence positive sur la volonté du personnel scolaire d'intégrer un enfant ayant un TSA. D'autres études rapportent également que la capacité de la direction à établir un climat de collaboration entre les membres du personnel scolaire tout en transmettant les valeurs inhérentes au respect de l'intégration scolaire est un déterminant de la réussite de l'intégration (Gibb, Tunbridge, Chua et Frederickson, 2007; lalongo, 2007; Segall et Campbell, 2014).

\section{L'enseignante}

Lorsqu'un enfant est intégré en milieu ordinaire, il côtoie quotidiennement son enseignante, ce qui fait de cette dernière l'une des principales actrices impliquées auprès de l'enfant. Pour cette raison, la perception des enseignantes concernant l'intégration scolaire est celle qui, jusqu'à présent, a été le plus largement documentée. De nombreuses recherches ont souligné le manque flagrant de formation spécifique et d'expérience avec les élèves ayant un TSA chez les enseignantes intégrant ces enfants. Malheureusement, ce manque de connaissance entraîne, le plus souvent, des conceptions et des croyances erronées concernant les méthodes d'intégration, les méthodes d'apprentissage et les caractéristiques du TSA (Helps, Newsom-Davis et Callias, 1999; Segall et Campbell, 2014; Siklos et Kerns, 2006; Unianu, 2012). Pourtant, les enseignantes considèrent qu'une formation spécifique au TSA est un facteur déterminant pour faciliter l'intégration de l'enfant (lalongo, 2007; Ruel, 2014). On peut donc se demander si les enseignantes ont les ressources nécessaires pour suivre ces formations.

La littérature a également mis en lumière le lien qui unit l'attitude qu'adopte l'enseignante vis-à-vis l'enfant intégré et ses connaissances relatives au TSA. À ce sujet, plusieurs études mettent en évidence que les enseignantes qui ont reçu une formation expliquant les caractéristiques du TSA sont beaucoup plus intéressées à accueillir ces enfants que ceux n'ayant reçu aucune formation (Dybvik, 2004; lovannone, Dunlap, Huber et Kincaid, 2003; McGregor et Campbell, 2001). Le 
manque de connaissance sur le TSA entraînerait également une mauvaise interprétation des comportements de l'enfant ce qui peut créer de l'exaspération chez l'enseignante (Emam et Farrell, 2009).

Outre le savoir, c'est-à-dire les connaissances, que l'enseignante peut acquérir sur le TSA, cette dernière doit également avoir une vision favorable de l'intégration pour accepter d'adapter son enseignement aux besoins de l'enfant (Ruel, 2014; Segall et Campbell, 2012). De plus, une attitude positive et de respect à l'égard de l'élève intégré de la part de l'enseignante peut également être un facteur déterminant dans l'acceptation des pairs vis-à-vis ce dernier. En effet, les autres enfants auront tendance à adopter les attitudes et les comportements que l'enseignante manifeste à l'endroit de l'élève ayant un TSA (Horrocks, White et Roberts, 2008).

\section{L'accompagnatrice}

Peu de recherches ont été menées sur l'influence des attitudes, des connaissances et des compétences des personnes accompagnant l'enfant présentant un TSA intégré en classe ordinaire. Pourtant, l'utilisation d'un accompagnement est l'une des mesures les plus utilisées dans un contexte d'intégration scolaire (Giangreco, Edelman, Broer et Doyle, 2001). Une étude menée auprès des personnes accompagnant l'élève a mis en lumière le fait que ces dernières considèrent qu'elles devraient bénéficier d'une meilleure formation spécialisée pour répondre adéquatement aux besoins de l'enfant (Eldar, Talmor et Wolf-Zukerman, 2010). Cette perception est confirmée par les propos de certaines enseignantes qui, malgré leur perception positive d'avoir une aide supplémentaire en classe, souligne qu'elles ne sont pas nécessairement satisfaites de la qualité des services reçus (Ruel, 2014). Qui plus est, au Québec, le rôle de l'accompagnatrice n'est pas clairement défini et varie beaucoup passant, selon les situations, d'un soutien axé sur les tâches académiques à un soutien lié la gestion du comportement de l'enfant (Paquet, Forget et Giroux, 2009).

À notre connaissance, aucune recherche québécoise n'a documenté le regard des mères sur les caractéristiques du personnel scolaire qui, selon elles, influencent l'intégration en classe ordinaire de leur enfant. Pourtant, plusieurs recherches montrent l'importance de documenter le point de vue de ces dernières (Courcy, 2014; des Rivières-Pigeon et Courcy, 2014; Granger, 2011; Sabourin, 2012). Notre posture théorique nous amène donc à questionner spécifiquement la perception des mères concernant les éléments qui ont pu influencer, selon elles, l'intégration en classe ordinaire de leur enfant présentant un TSA.

\section{Objectif de l'étude}

À la lumière des résultats des recherches effectuées jusqu'à maintenant, il apparaît important de mieux documenter la perception des mères québécoises à propos des éléments qui, selon elles, ont influencé le déroulement de l'intégration scolaire en classe ordinaire de leur enfant présentant un TSA. Cet article, de nature exploratoire, abordera de façon spécifique trois aspects de cette perception : ceux qui concernent les attitudes, les compétences et les connaissances sur le TSA 
du personnel scolaire travaillant auprès de l'enfant présentant un TSA. Puisqu'il s'agit d'une recherche effectuée avec une méthodologie qualitative, notre objectif n'est pas de générer des résultats généralisables à l'ensemble des familles dont l'enfant présentant un TSA est intégré en classe ordinaire, mais plutôt d'analyser l'expérience décrite par les 18 mères rencontrées afin de mieux comprendre la réalité qu'elles et leur enfant ont vécue.

\section{Méthode}

\section{Participantes}

Dans le cadre de cette recherche, effectuée avec une méthodologie qualitative, dix-huit entretiens ont été conduits auprès de mères québécoises d'enfant présentant un TSA intégré en classe ordinaire au 1er ou 2ème cycle du primaire. Puisqu'elles ont toutes démontré un intérêt pour participer à l'étude, aucune n'a été exclue. Dix-sept des dix-huit mères sont soit mariées $(n=12)$ soit en union de fait $(n=5)$ et une mère vit seule. La scolarité des mères est variée passant du niveau secondaire $(n=3)$, collégial $(n=8)$ à universitaire $(n=7)$. Six ont un emploi à temps plein, six un emploi à temps partiel, trois sont mères au foyer, deux sont en congé non rémunéré et une est étudiante. Dans les familles interrogées, trois ont un enfant $(16,7 \%)$, neuf ont deux enfants $(50 \%)$, cinq ont trois enfants $(27,8 \%)$ et une a cinq enfants $(5,6 \%)$. Trois des enfants présentant un TSA $(16,7 \%)$ avaient une sœur ou un frère avec le même diagnostic.

Certaines caractéristiques sont homogènes chez les enfants ayant un TSA dont les mères ont participé à l'étude. Aucun ne présente une déficience intellectuelle et tous ont un diagnostic de TSA représenté, selon la nomenclature du DSM-IV-TR, par de l'autisme $(n=4)$, de l'Asperger $(n=7)$ ou un TED-NS $(n=7)$. Nous avons maintenu les catégories diagnostiques du DSM-IV, car au moment des entretiens, celles-ci étaient encore en vigueur. Parmi les 18 enfants, douze n'ont pas de diagnostic associé. Par contre, des diagnostics comorbides étaient présents chez certains enfants comme le TDA/H $(n=3)$, la dyspraxie $(n=2)$, l'anxiété $(n=2)$, I'hypotonie $(n=1)$, la déficience du système immunitaire $(n=1)$, la scoliose $(n=1)$ et le trouble de la modulation sensorielle $(n=1)$. Les enfants sont intégrés au sein d'écoles provenant de 10 commissions scolaires francophones à travers la province de Québec. Treize enfants ont fréquenté une seule école depuis le début de leur scolarisation et cinq en ont fréquenté deux.

\section{Procédures}

Les mères ont été sollicitées par l'entremise de divers organismes, associations, cliniques, commissions scolaires et réseaux sociaux. Une personne responsable dans chaque organisation a remis aux mères répondant aux critères d'inclusion, une lettre leur expliquant le projet et leur possibilité de participer à l'étude. Certains organismes ont préféré afficher l'annonce de recrutement sur leur site internet avec les lignes directrices du projet. Afin de respecter un consentement libre, ce sont les mères intéressées à participer à l'étude qui ont contacté l'expérimentatrice pour obtenir de plus amples informations. 
Les entretiens se sont déroulés au cours de l'année 2011-2012. Les mères ont contacté la personne responsable du projet et une première étape de sélection a été effectuée afin de nous assurer que toutes les répondantes présentaient un profil respectant les critères d'inclusion de l'étude et que leur consentement était donné de manière libre et éclairée. Ce premier contact a également été l'occasion de prendre un rendez-vous et de choisir un lieu de rencontre (local à l'UQAM, domicile, bibliothèque, Skype, etc.) à leur convenance. Parmi les mères qui nous ont contactées, trois habitaient en région éloignée. Afin de permettre à ces mères de participer à la recherche et afin d'obtenir un échantillon plus diversifié, nous avons accepté de faire les entretiens à l'aide de l'application informatique Skype. Cette méthode de collecte de données est encore peu utilisée en recherche. Son utilisation a permis de constater que le contact vidéo constituait un médium convivial avec lequel les mères se sont dites très à l'aise. Ces entrevues ont permis d'obtenir des informations aussi riches que celles recueillies auprès des mères que nous avons rencontrées en personne. Les participantes étaient rencontrées une seule fois pour une durée variant entre 90 et 120 minutes. Tous les entretiens ont été enregistrés sur un enregistreur numérique et ont par la suite été retranscrits intégralement aux fins d'analyse.

Le guide d'entretien a été élaboré par la responsable de la recherche à partir de la littérature et de la révision du contenu par des experts du domaine du trouble du spectre de l'autisme et du milieu de l'éducation. Au final, six sections ont été retenues. Elles portaient sur : (1) les services de préscolarisation, (2) la transition vers l'entrée à l'école primaire, (3) l'intégration, (4) l'influence de l'intégration scolaire sur l'enfant présentant un TSA, (5) l'influence de l'intégration scolaire sur la mère et la famille et (6) la poursuite de la scolarisation de leur enfant. Ce guide étant ouvert, il fut adapté au fil des entretiens pour permettre d'obtenir le plus d'information possible (voir Annexe 1).

\section{Traitement et analyse des données}

Nous avons privilégié une analyse qualitative thématique telle que décrite par Braun et Clarke (2006) dont l'objectif est d'identifier et d'analyser des thèmes émergeant du contenu des entretiens. En psychologie, l'analyse thématique est fréquemment utilisée pour explorer des sujets peu documentés ce qui correspond aux besoins de la présente recherche. Nous avons en effet remarqué, au cours de l'analyse, que plusieurs thèmes qui n'étaient pas abordés dans les recherches que nous avons recensées ont émergé. II était donc pertinent de privilégier une approche nous permettant de traiter l'ensemble de l'information contenue dans les entretiens.

Le cadre proposé par Braun et Clarke (2006) est de nature inductive, c'est-à-dire que la thématisation et la catégorisation ne sont pas imposées en fonction d'un cadre théorique, mais émerge du discours des mères rencontrées. Pour réaliser l'analyse, nous avons suivi les six (6) étapes proposées par ces auteurs. Pour faciliter la classification et l'analyse des données, le logiciel NVivo a été utilisé. Un codage "libre » en unité de sens des transcriptions a été effectué par la responsable de la recherche. Chaque unité de sens représente une idée unique qui peut se traduire en un mot, une phrase ou un paragraphe. Cette étape 
a permis de collecter une grande quantité d'unité de sens qui ont été, par la suite, classifiés dans des thèmes potentiels (par ex. toutes les unités de sens évoquant l'implication de la mère ont été classifiées ensemble). À ce moment, des catégories et des sous-catégories ont été élaborées afin de bien définir chaque thème. Cette procédure a permis de constituer une carte des principaux thèmes, concepts-clés, et des principaux liens qui s'établissaient entre ces derniers.

\section{Résultats}

Notre objectif était d'identifier ce qui, selon les mères, influence le déroulement d'intégration scolaire de leur enfant. Comme mentionné précédemment, notre démarche de recherche était inductive : nous avons laissé les mères s'exprimer sur les facteurs qu'elles considèrent être des éléments clés pour l'intégration de leur enfant. Le propos des mères a clairement fait ressortir que les caractéristiques du personnel scolaire sont considérées comme des éléments déterminants de l'intégration de leur enfant. II est toutefois important de garder à l'esprit que tous les résultats présentés décrivent la perception des mères et non une mesure directe des attitudes, des compétences et des connaissances sur le TSA du personnel travaillant auprès de l'enfant.

Avant de décrire de façon précise les différents éléments qui ont émergé de l'analyse, il importe de souligner un premier constat qui s'est dégagé de façon marquée du propos des mères rencontrées. En effet, nous avons constaté que chacune des caractéristiques du personnel scolaire qui se sont révélées facilitantes, selon les mères, pour l'intégration de leur enfant, ont également été identifiées par ces mêmes mères ou par d'autres comme une source de difficulté, lorsque ces caractéristiques étaient absentes. Par exemple, la présence d'une expertise, de la part de l'enseignante, concernant les caractéristiques du TSA était identifiée à la fois comme une caractéristique facilitant l'intégration, lorsque cette expertise était présente, et un obstacle à celle-ci, lorsqu'elle était absente. Pour cette raison, nous exposerons ensemble les caractéristiques perçues par les mères comme étant positives et négatives, car celles-ci étaient généralement le miroir l'une de l'autre. Un second constat ressort des résultats de notre étude : les attitudes, les connaissances sur le TSA et les compétences des personnes travaillant auprès de l'enfant sont des facteurs qui sont souvent liés. Ce résultat correspond à ce que d'autres auteurs ont constaté (Dybvik, 2004; lovannone, Dunlap, Huber et Kincaid, 2003; McGregor et Campbell, 2001). Nous exposerons donc dans la description des résultats les diverses relations présentes entre les catégories. Par exemple, nous décrirons comment les connaissances sur le TSA des membres du personnel scolaire ont, selon les mères, influencé l'attitude de ces derniers à l'égard de l'enfant.

Outre ces constats généraux, l'analyse des propos des mères a fait émerger deux (2) grandes catégories de caractéristiques du personnel scolaire qui ont influencé le déroulement de l'intégration scolaire de leur enfant : (1) l'attitude du personnel scolaire et (2) leurs compétences et connaissances sur le TSA. 
L'analyse du propos des mères met en évidence qu'une importance particulière est accordée aux comportements et aux attitudes que le personnel adopte avec elles et leur enfant. La majorité des mères ont vécu des expériences qu'elles jugent négatives dans leurs relations avec les membres du personnel scolaire (direction, enseignante, TES, accompagnatrice ou professionnel). Pour la plupart, les attitudes ou les comportements dont il est question dans ces propos impliquent un manque d'ouverture, d'écoute, de coopération/de collaboration, d'implication, ou encore une banalisation des difficultés de l'enfant. Soulignons ici que les mères qui ont vécu des expériences négatives avec certains membres du personnel rapportent également des expériences positives avec d'autres. On retrouve donc une grande diversité d'expériences au sein d'une même famille, les relations avec le personnel pouvant présenter d'importantes variations d'année en année en fonction de l'environnement scolaire.

\section{Démontrer de l'empathie et offrir du soutien}

Plusieurs mères ont dit avoir apprécié que le personnel scolaire (direction, enseignante, et TES/accompagnatrice) se soit investi personnellement auprès d'elles et de leur enfant, au-delà de ce qui était nécessaire sur un plan strictement professionnel. En effet, dans les cas où l'intégration a été perçue positivement, les mères ont longuement parlé de la relation qu'elles avaient développée avec le personnel. Certaines ont particulièrement apprécié le soutien émotionnel apporté par des enseignantes qui, même dans un contexte de situation difficile avec l'enfant en classe, ont continué à valoriser les mères en leur faisant part de leur appréciation de leurs compétences parentales.

Une autre forme de soutien évoquée par les mères est le fait que des membres du personnel scolaire puissent être présents pour les accompagner lors des démarches nécessaires à l'évolution de leur enfant en milieu scolaire. Par exemple, une directrice d'école sensible aux difficultés vécues par une mère a offert du soutien en étant présente et en faisant un suivi régulier des services offerts à l'enfant : "Dans les premières semaines, elle est venue me voir pour me demander si tout se passait bien. Lorsqu'il y a eu un changement d'éducatrice pour la supervision au niveau de l'entrée et des sorties, elle est venue me la présenter. Elle voyait que j'étais inquiète $»^{4}$ (Mère \#18). Une autre mère explique que ses discussions informelles avec l'accompagnatrice du service de garde lui ont permis de voir sa situation quotidienne autrement : "Elle (en parlant d'un accompagnateur du service de garde) a un enfant qui est autiste. Alors, je me suis dit : "on parle le même langage"'. Je me disais qu'ils allaient penser qu'il fait des crises parce qu'il vit des problèmes à la maison, mais elle m'a dit “'Non, pour ma fille, c'est pareil"' " (Mère \#05).

4. La forme des extraits de verbatims a été légèrement modifiée afin d'en faciliter la lecture. 


\section{Respecter la différence des enfants}

Un autre constat intéressant a émergé du propos des mères: les enseignantes qui ont adopté une attitude d'ouverture ont créé un climat de classe empreint de ce respect, ce qui aurait eu, un effet positif sur le regard que posent les autres enfants sur l'enfant intégré. Ainsi, le fait que l'enseignante ou l'accompagnatrice choisisse d'expliquer les particularités de l'enfant aux autres élèves, et ce, sans le stigmatiser, a été, selon plusieurs mères, très aidant pour l'acceptation de l'enfant par ses pairs. Comme en témoigne cette mère : " (l'enfant) devait porter des coquilles dans certains cours; (l'enfant) a été devant la classe expliquer pourquoi il portait des coquilles et il a dit qu'il avait des oreilles supersoniques. Après, dans sa classe, tout le monde voulait mettre des coquilles comme (l'enfant). Je pense que cela fait une grosse différence " (Mère \#04). Dans certains cas, les mères ont également dit qu'afin d'éviter la stigmatisation de leur enfant, certaines adaptations ont été offertes à tous, permettant ainsi à l'enfant de se sentir comme tous ses camarades de classe : " J'ai aimé ça, cette façon de le faire. Ils ne faisaient pas une adaptation juste pour (l'enfant), ils le faisaient pour toute la classe. Ils ont mis des photos et ils ont expliqué à tout le monde à quoi ça servait. Mais en fait, c'était pour (l'enfant), parce qu'il se sauvait » (Mère \#14). Ce témoignage montre à quel point l'attitude du personnel scolaire joue un rôle dans l'acceptation de l'enfant par ses pairs et offre l'occasion aux autres enfants de développer à leur tour un regard positif sur l'enfant. En revanche, dans un environnement où les autres élèves ne sont pas " préparés " à côtoyer un élève qui a besoin d'adaptation, ceci peut créer des questionnements embarrassants pouvant même perturber émotionnellement l'enfant: "Tous les autres amis ne comprenaient pas pourquoi (l'enfant) avait un ordinateur portable. Ils ont bombardé (l'enfant) de questions, et comme (l'enfant) se sentait regardé, il s'est fâché, et tout a revolé. Ça aurait été pourtant si simple de les préparer, mais ça n'a pas été fait » (Mère \#09). Ici encore, les mères considèrent que l'attitude du personnel scolaire constitue un élément crucial pour le développement de relations harmonieuses entre l'enfant intégré et les adultes, mais également avec les autres enfants.

\section{Reconnaître le rôle et l'expertise des mères}

Le contenu des entretiens met en évidence que les mères désirent être impliquées dans l'éducation de leur enfant et qu'elles veulent être sollicitées pour prendre des décisions pouvant influencer l'avenir de ce dernier. Selon les mères, le personnel scolaire qui a adopté une attitude d'ouverture et d'écoute à leur égard était plus enclin à les interpeller pour obtenir des conseils ou avoir leur approbation, les reconnaissant ainsi comme un "parent-expert» de leur enfant. Comme le montre l'extrait suivant, selon les mères, cette reconnaissance du " parent-expert » permet à l'équipe-école de mieux comprendre la détresse de l'enfant et d'intervenir de façon appropriée: "On avait une belle communication pour faire le suivi. Elle était très à l'écoute des conseils que l'on donnait. Je disais : " regarde, nous quand il fait ça, on agit de telle façon, et ça le calme ou ça l'aide à comprendre ». Elle disait : " Je vais essayer ça ». On partageait nos trucs, là » (Mère \#11). Le fait que l'équipe-école reconnaisse l'expertise de la mère, notamment en ce qui a trait aux particularités de son enfant, permet non seulement de valoriser le rôle des mères 
dans l'éducation de leur enfant mais également de mettre en place des interventions qui correspondent à ses besoins.

Le désir du personnel scolaire d'impliquer activement les mères dans les rencontres de plans d'intervention était apprécié par ces dernières, car elles considèrent qu'il est important qu'elles puissent donner leur point de vue sur les besoins et les moyens à privilégier pour aider leur enfant. Les mères qui avaient le sentiment que l'école travaillait en continuité avec les interventions réalisées à la maison sentaient que l'école était davantage en mesure d'aider l'enfant. Malheureusement, les propos de plusieurs mères montrent au contraire que l'expertise et la connaissance qu'elles ont de leur enfant ne sont pas toujours prises en considération. Celles-ci étaient même parfois ignorées, lors de rencontres avec le personnel ou pour l'élaboration du plan d'intervention: "Le problème c'était qu'eux, ils donnaient leurs idées, moi j'avais les miennes, mais les miennes n'étaient pas mises dedans (en parlant du plan d'intervention). " (Mère \#14). Ce type d'expérience a laissé un goût amer, donnant aux mères l'impression que les besoins de leur enfant ainsi que les leurs n'étaient pas pris en considération.

\section{Ne pas minimiser les difficultés de l'enfant}

L'analyse du propos des mères révèle que la façon dont les membres du personnel scolaire perçoivent les besoins particuliers de l'enfant aurait, une influence directe sur la mise en place de moyens pour aider celui-ci. En effet, nous avons remarqué que lorsque les mères percevaient que le personnel scolaire était attentif aux particularités de l'enfant, il était beaucoup plus fréquent d'assister à une mise en place rapide de mesures visant à répondre aux besoins de ce dernier. Cette attitude, que nous pouvons qualifier de "proactive », permettait, selon les mères, de sécuriser l'enfant en répondant à ses besoins et de faire en sorte qu'elles se sentent en confiance avec l'école sachant que les difficultés vécues par leur enfant seraient prises en considération. L'extrait suivant montre à quel point les mères peuvent se sentir soulagées lorsqu'elles perçoivent que l'école a su détecter immédiatement que l'enfant aurait besoin d'une aide adaptée : «À partir de ce moment-là la directrice m'a appelée. Tout de suite, elle a regardé ce qu'on pouvait faire pour faire baisser l'anxiété (de l'enfant), et non pas combien de jours je pouvais le garder pour qu'il se sente mieux avant de revenir » (Mère \#07).

En revanche, le fait de minimiser les difficultés de l'enfant, une attitude fréquemment dénoncée par les mères rencontrées, est perçu comme menant à une exacerbation des comportements problématiques de l'enfant ce qui nuit à l'évolution de celui-ci au sein du milieu scolaire. En effet, lorsque les mères perçoivent que les difficultés de l'enfant sont minimisées, cela aurait non seulement pour effet de nuire à l'obtention de services, mais également de faire vivre aux mères des moments difficiles d'impuissance et de désespoir.

À l'inverse, lorsque le personnel n'agissait pas de manière proactive, les mères, choisissaient d'adopter une posture plus revendicatrice, pour que les besoins particuliers de leur enfant soient pris en considération. Plusieurs d'entre elles ont tenté de faire valoir les besoins particuliers de préparation, de stabilité de l'environnement ou de l'importance de recevoir des services auprès du personnel 
scolaire, mais souvent en vain. Malheureusement, les propos des mères met en évidence que dans de nombreux cas, plus celles-ci tentaient de revendiquer des services pour leur enfant plus les relations avec le personnel scolaire devenaient tendues.

\section{Adopter une attitude de collaboration}

Selon les mères, l'attitude adoptée par la direction, non seulement envers les mères, mais également envers les autres membres du personnel scolaire (enseignante, TES et professionnels) a une influence notable sur l'expérience d'intégration de leur enfant. En effet, plus la direction adopte une posture " hiérarchique ", plus les mères cherchaient à revendiquer des services pour leur enfant, et plus il s'est créé un climat de méfiance entre la mère et la direction de l'école. La mère qui s'exprime dans l'extrait suivant considère que climat tendu entre la direction et le personnel scolaire l'a empêchée d'obtenir les services nécessaires à son enfant: "Quand elle demandait dans la classe si tout était correct, ils lui disaient que tout allait bien (en parlant de la directrice et du personnel de la classe). Je lui ai dit que non, ça n'allait pas si bien (en parlant d'une conversation entre la mère et la direction). Ce n'est pas la directrice, c'est plutôt la collaboration avec la professeure et l'éducatrice " (Mère \#18).

Au contraire, les écoles où la relation entre la direction et les membres du personnel scolaire était empreinte de respect, les mères estimaient qu'elle et leur enfant étaient accueillis et que les besoins de ce dernier étaient au cœur des préoccupations du personnel : "C'est des gens qui travaillent bien ensemble (en parlant du personnel scolaire et de la direction). Quand j'ai des questions, quand j'arrive au plan d'intervention, je n'ai pas l'impression qu'ils ne se sont jamais vus, qu'ils ne se sont jamais parlés, qu'ils ne sont pas d'accord. Je n'ai pas senti ça, tout le monde allait à la même place, tout le monde avait le même objectif que moi pour (l'enfant) » (Mère \#7). Cet extrait suggère que la qualité de la collaboration entre le personnel de l'école a créé un climat favorable à l'intégration de l'enfant. D'ailleurs, seules les mères qui ont décrit la collaboration entre les membres du personnel comme étant positive ont été sollicitées par ces derniers pour s'impliquer au sein de l'école pour aider à répondre aux besoins de l'enfant.

Ainsi, ce que les mères considèrent comme un manque de soutien de la part de la direction ou de la commission scolaire envers leurs enseignantes et leurs professionnelles était jugé comme ayant des impacts sur la qualité des services offerts aux enfants. Certaines mères croient qu'il serait utile de fournir une aide supplémentaire en tout temps dans la classe, d'autres proposent que le nombre d'élèves par classe soit diminué pour faciliter le travail du personnel. Le manque de soutien constaté par les mères serait, selon ces dernières, responsable de surmenage chez le personnel scolaire, ce qui expliquerait, toujours selon elles, des départs précipités. Cette situation instable créée par un roulement de personnel évitable serait néfaste pour l'enfant, car celui-ci a besoin de routine et de prévisibilité. Le tableau 1 résume les principales catégories qui ont émergé de l'analyse pour cette première section. 
Tableau 1. Attitudes du personnel scolaire qui influencent le déroulement de l'intégration

\begin{tabular}{ll}
\hline Attitudes & Extrait d'entretien illustrant la catégorie \\
\hline
\end{tabular}

Démontrer de l'empathie et offrir du soutien

Respecter la différence des enfants

Reconnaître le rôle et l'expertise des mères

Ne pas minimiser les difficultés de l'enfant

Adopter une attitude de collaboration
« Moi, le premier bulletin m'a anéantie, il a confirmé toutes mes craintes. Elle a été très soutenante aussi pour nous, pour nous dire que nous étions des bons parents. Juste ça, ça m'a fait du bien. Je n'aurais pas eu besoin de quelqu'un qui m'aurait fait sentir que je faisais les choses pas correctement » (Mère \#13).

" II y avait une notion de respect là-dedans aussi et d'ouverture de la part autant de l'équipe qui a fait comprendre ça aux enfants » (Mère \#09).

" Ils étaient toujours à l'écoute, ils demandaient toujours notre avis avant de commencer quelque chose " (Mère \#16).

" Le problème a duré très longtemps, parce que l'école ne le prenait pas au sérieux. Ils minimisaient beaucoup le problème. Quand je leur disais que mon fils faisait des crises, épouvantables, ils disaient : ce n'est pas grave. Ça a pris longtemps avant qu'ils agissent » (Mère \#01).

« II manque de soutien à l'enseignement comme aux TES. On peut blâmer les enseignants de ne pas vouloir accueillir nos enfants, mais il n'y a pas de soutien en classe " (Mère \#04).

\section{Les compétences et les connaissances sur le TSA du personnel scolaires}

La deuxième catégorie de caractéristiques qui, selon les mères, influence le déroulement de l'intégration scolaire de leur enfant, concerne les connaissances et les compétences qu'elles perçoivent chez ceux-ci. Ce qui distingue cette section de la précédente est qu'il s'agit de caractéristiques qui ne sont pas forcément reliées à leur l'attitude adoptée envers les mères et les enfants. En effet, même parmi les mères qui ont développé de bonnes relations avec des membres du personnel, plusieurs déplorent malgré tout le manque de connaissance de ce personnel concernant le TSA et la façon d'intervenir auprès de ces enfants. Toutefois, les mères qui décrivaient cette situation faisaient généralement preuve d'une grande compréhension face au personnel concerné : "Ils ont géré ses comportements comme des troubles de comportement, au lieu de le gérer comme du TSA. Je pense que ça a envenimé les choses, mais ce n'était pas de la mauvaise volonté, c'était simplement de la méconnaissance » (Mère \#09). Soulignons que la majorité des mères rencontrées ont été confrontées au manque de connaissances sur le TSA de la part des personnes travaillant auprès de l'enfant. 


\section{Connaître et comprendre les besoins de l'enfant}

Les mères rencontrées estiment que le manque de connaissance sur le TSA a souvent fait en sorte que le personnel, préscolaire et scolaire, n'a pas été en mesure d'identifier de façon précoce les symptômes dus au TSA de leur enfant. Elles jugent en effet que cette situation a eu pour conséquence, d'une part, d'empêcher que leur enfant ait accès à des services et, d'autre part, de mener à une mauvaise compréhension des besoins de celui-ci. Selon plusieurs mères, l'absence de détection précoce du TSA chez leur enfant a influencé la qualité de la transition vers l'école puisque plusieurs de ces enfants n'ont pas pu bénéficier d'une préparation adéquate pour cette étape (par ex. atelier préparatoire, acquis de motricité fine, apprentissage des règles sociales, etc.). À ce sujet, la hausse des comportements problématiques lors de l'entrée à l'école (par ex. anxiété, colère, crises, etc.) et les difficultés d'adaptation sont considérées par plusieurs mères comme étroitement liées au fait que leur enfant n'aurait pas été en mesure de se préparer au nouvel environnement.

Des mères ont également déploré le fait que, selon elles, le personnel scolaire éprouve souvent des difficultés à cibler les besoins particuliers de leur enfant, surtout lorsque ceux-ci sont attribuables au TSA. Elles jugent donc que la qualité des plans d'intervention est souvent discutable puisqu'ils doivent être basés sur l'identification des besoins de leur enfant. Même lorsque l'enfant a bénéficié d'un plan d'intervention adéquat, certaines mères estiment que les difficultés de leur enfant ont été minimisées en raison de "l'invisibilité » du TSA. Elles considèrent également que ce qui est ici qualifié d'un manque de compréhension, de la part du personnel, des particularités liées à cette problématique a mis en péril la mise en place des adaptations pour l'enfant.

En revanche, les mères qui considèrent que le personnel scolaire a été en mesure d'observer et de comprendre l'enfant avaient une vision beaucoup plus positive de son intégration. Dans l'extrait suivant, une mère raconte comment les besoins de son enfant ont été ciblés rapidement grâce au travail d'observation de l'accompagnateur : "Elle a vraiment été capable de faire un travail d'observation (en parlant de l'accompagnateur) qui lui a permis de venir avec des éléments concrets" (Mère \#08). Un autre aspect soulevé par les mères concerne l'importance accordée, par le personnel scolaire, à la recherche de l'origine des difficultés de l'enfant. En effet, selon les mères, les écoles qui ont adopté une attitude proactive dans la recherche de solutions pour l'enfant ont réussi à l'aider en lui montrant des comportements alternatifs : "Sur le moment, ils font ce qu'il faut pour éviter la crise, mais après ça, ils disent : ça c'est une situation qui le met en crise, donc on retravaillait les outils pour l'aider à gérer sa prochaine crise " (Mère \#11). Plusieurs mères considèrent que les enfants qui ont fréquenté une école où le personnel n'avait pas développé le réflexe de chercher l'origine du comportement, les situations problématiques avaient tendance à se créer et à perdurer : " Mais ils ne vont pas chercher à la source. Ils envoient le carnet et c'est fini » (Mère \#15). 


\section{Offrir des interventions adaptées aux besoins de l'enfant}

Comme nous l'avons vu dans la section précédente, les mères estiment que le fait d'avoir des difficultés à identifier les besoins de leur enfant nuit à la qualité des interventions. II en est de même pour le manque d'expérience de certaines intervenantes qui également peut influencer la qualité de l'intervention. Devant ces difficultés, certaines mères ont tenté de proposer des solutions à l'enseignante et à l'accompagnatrice. Par contre, elles constataient souvent que celles-ci n'étaient pas en mesure de les appliquer adéquatement : «On pouvait suggérer des choses, mais elle n'était pas capable de les mettre en pratique. Donc, elle avait de bonnes intentions, mais elle ne le faisait pas. On a appris a (l'enfant) à aller la voir quand il avait besoin, mais il n'y avait pas de suite. Après un moment, il ne lui demandait plus » (Mère \#04).

Si certaines mères ont parlé de l'inexpérience ou du manque de compétence du personnel scolaire, d'autres ont été confrontées à des façons de faire qu'elles ont jugées inacceptables et qui les ont profondément choquées. Dans certains cas, ce type d'intervention a mené à de graves colères chez l'enfant, qui ont non seulement nui à l'établissement d'un lien de confiance entre l'enfant et le personnel scolaire, mais ont également mis fin à l'intégration d'un enfant, qui a été expulsé de la classe ordinaire. Par exemple, certaines interventions s'apparentaient, selon les mères, à des « menaces » pour l'enfant, ce qui a eu pour effet de le désorganiser complètement : « La TES l'a menacé de briser son bracelet en scoubidou. Elle a fait semblant de lui couper et il s'est mis à hurler. " (Mère \#01). De plus, lorsque les mères insatisfaites ont tenté de s'impliquer pour améliorer la qualité des interventions, celles-ci ont trouvé que l'école n'a pas été réceptive à leurs suggestions. Dans le cas d'un enfant, la mise en place d'interventions perçues inappropriées par la mère, a mené à des problèmes si importants que l'enfant a été suspendu de l'école de façon répétée avant d'être exclu du régulier : " (L'enfant) était retiré, pratiquement tous les jours. Ça ne faisait pas une heure qu'il était à l'école et on m'appelait pour me le retourner à la maison. II a eu 18 suspensions » (Mère \#09).

Les mères satisfaites de la qualité des interventions du personnel scolaire ont plutôt évoqué les qualités d'encadrement ou de soutien du personnel vis-à-vis l'enfant, ce qui a permis à ce dernier de développer sa confiance et son autonomie : "L'encadrement dans les transitions, diminuer son stress, l'empêcher de s'éparpiller, le valoriser, lui montrer qui était capable de faire certaines tâches que je pense qu'il se serait découragé s'il avait été laissé à lui-même » (Mère \#13). En effet, l'analyse des propos permet de constater que plus les mères considèrent que les interventions du personnel scolaire sont de bonne qualité, plus elles perçoivent positivement l'évolution de leur enfant dans le contexte d'intégration. L'analyse du propos des mères montre également que les mères satisfaites de l'intégration mettent en évidence que l'enseignante ou l'accompagnatrice a su adapter le matériel que l'enfant utilise afin de faciliter les apprentissages de ce dernier. Par exemple, une enseignante qui avait compris qu'un élève ayant un TSA avait besoin de prévisibilité a instauré un endroit pour lui afin qu'il puisse toujours avoir un repère constant dans la classe : "L'enseignante lui a fait un petit coin dans la classe. Donc (l'enfant) était tout le temps à la même place dans la classe, contrairement aux autres enfants " (Mère \#09). II est également clair que le fait de bien comprendre les effets du TSA 
sur le quotidien de l'enfant a, selon les mères, permis au personnel de mieux cibler les besoins, mais également les moyens d'interventions les plus adéquats tout en respectant les limites de l'enfant: " Je veux qu'elle l'amène au même niveau que les autres, sauf qu'elle est capable de voir les difficultés de (l'enfant) et d'en tenir compte » (Mère \#17).

\section{Quand ces manques perçus poussent les mères à former le personnel}

Nous avons vu dans la section précédente que plusieurs mères perçoivent les interventions offertes dans le milieu scolaire comme inadéquates, notamment parce que le personnel scolaire manque de connaissances ou de formation concernant le TSA. Plusieurs mères ont donc décidé de pallier à ce manque d'expertise en allant chercher elles-mêmes l'information auprès de professionnels. Certaines, par exemple, ont assisté à des formations sur le TSA dans le but de transmettre certaines notions au personnel scolaire souhaitant que celui-ci les mette en application.

Dans certains cas, l'école ne semblait même pas en mesure de fournir le matériel de base pour intervenir auprès de l'enfant et demandait à la mère de le préparer. Par exemple, selon un plan d'intervention, un enfant avait besoin de la mise en place de pictogrammes et d'un horaire. Comme l'école n'était pas en mesure de satisfaire ces besoins, la mère a été interpelée. On aura compris que ce type d'implication peut parfois être lourd pour les parents : « Un moment donné, je dis je ne peux ne pas faire les pictogrammes pour vous autres, je ne peux pas vous dire c'est quoi l'horaire qui va être parfait pour vous autres, c'est à vous de trouver. Vous avez une éducatrice, elle supposée savoir quoi faire » (Mère \#18).

D’autres mères sont allées jusqu'à « former » le personnel en leur montrant directement les techniques d'intervention: "Moi, il aurait fallu que j'y aille tous les jours et que je leur montre comment travailler avec mon garçon » (Mère \#18). Dans certaines écoles, la direction a accepté de libérer les intervenantes pour une rencontre de formation avec la mère de l'enfant. Si cette ouverture de la part de l'école a été appréciée par certaines mères, d'autres déplorent le fait que leur implication à l'école soit tenue pour acquise. Comme l'indique une mère dans l'extrait suivant, le fait qu'elle " compense » le manque de connaissance des intervenantes est, selon elle, tellement tenu pour acquis qu'elle se sent contrainte de poursuivre cette implication chaque année, l'école n'envisageant pas d'adopter de solution alternative, comme recourir à des professionnelles : " Je suis contente d'avoir eu la chance de le faire (en parlant de former les intervenants). Surtout avec le manque de services de l'école. Par contre, je trouve que parce que je le fais, l'école ne trouve pas les moyens de faire autrement. Les services ne se développent pas. II y a une dépendance qui se fait sur le support que je donne à (l'enfant) » (Mère \#04). Notons toutefois que ces mères soulignent qu'elles préfèrent être sollicitées par l'école, malgré l'épuisement éventuel que cette implication peut créer, plutôt que de laisser le personnel sans ressource et l'enfant sans service adéquat. Le tableau 2 illustre les principales catégories pour cette deuxième section 
Tableau 2. Les compétences et les connaissances sur le TSA du personnel scolaire Compétences et Connaissances Extrait d'entretien illustrant la catégorie
sur le TSA

Connaître et Comprendre les besoins de l'enfant

Offrir des interventions adaptées aux besoins de l'enfant

Quand ces manques perçus poussent les mères à former le personnel
« Ça été difficile de leur faire comprendre. Elle (en parlant d'une autre élève) a besoin de son fauteuil, et lui, il a besoin de ses repères, ses pictogrammes, sa routine». (Mère \#18)

« La TES avait un manque flagrant d'expérience.

Elle commençait. C'est dommage, elle était complètement dépourvue. Elle m'appelait, mais elle n'avait aucune solution». (Mère \#09)

« Chercher des choses sur l'anxiété, consulter une psychologue dans le privée, après aller rencontrer la psychologue à l'école. On veut les informer finalement : informer et former les gens qui sont à l'école». (Mère \#04)

\section{Discussion}

L'objectif de cet article était de documenter la perception des mères québécoises à propos des caractéristiques du personnel scolaire qui, selon elles, ont influencé le déroulement de l'intégration en classe ordinaire de leur enfant présentant un TSA.

L'analyse du propos des mères met en évidence que ces dernières considèrent les attitudes, les compétences et les connaissances sur le TSA de tous les membres du personnel scolaire, en particulier, de la direction, des enseignantes et des accompagnatrices comme des éléments influençant le déroulement l'intégration scolaire de leur enfant. Nos résultats sont donc concordants avec les études portant sur la perception des parents, canadiens, européens et américains (Brewin, Renwick et Schormans, 2008; Cappe, 2012; Renty et Roeyers, 2006; Siklos et Kerns, 2006; Starr et Foy, 2012; Starr, Foy, Cramer et Singh, 2006; Stoner, Angell, House et Bock, 2007; Stoner et al., 2005) ainsi qu'avec l'étude québécoise ayant porté sur une population présentant des incapacités multiples (Rousseau, Dionne, Vézina et Drouin, 2009) quant à l'importance qu'accordent les parents aux caractéristiques du personnel scolaire dans l'intégration en classe ordinaire de leur enfant présentant des besoins particuliers. Plusieurs des résultats recoupent également ceux recueillis auprès de membres du personnel scolaire particulièrement en ce qui concerne l'importance pour ces derniers d'avoir des connaissances sur le TSA avant d'intégrer ces enfants (Eldar, Talmor et Wolf-Zukerman, 2010; Helps, Newsom-Davis et Callias, 1999; Horrocks, White et Roberts, 2008; Segall et Campbell, 2014; Siklos et Kerns, 2006; Symes et Humphrey, 2011; Unianu, 2012) et d'adopter une attitude d'ouverture face à l'intégration scolaire de ces enfants 
(Dybvik, 2004; Emam et Farrell, 2009; Gibb, Tunbridge, Chua et Frederickson, 2007; lalongo, 2007; lovannone, Dunlap, Huber et Kincaid, 2003; McGregor et Campbell, 2001, Segall et Campbell, 2014). Ces conclusions montrent que les caractéristiques des personnes travaillant auprès de l'enfant présentant un TSA représentent un aspect central du déroulement l'intégration scolaire de ces enfants.

Nos résultats, comme ceux des autres études mentionnées précédemment, suggèrent que l'attitude adoptée par la direction, les enseignantes et les accompagnatrices envers l'enfant et ses parents joue un rôle central dans les perception des parents eu égard à l'intégration de leur enfant. De façon plus spécifique, nos résultats corroborent ceux de Stoner et al. (2005) selon lesquels les mères devraient être considérées comme des membres à part entière de l'équipeécole, en ce qui concerne les décisions qui touchent leur enfant. Toujours selon ces auteurs, l'expertise qu'elles ont acquise au sujet du TSA et de leur enfant devrait être mise à profit. Une telle approche donnerait aux mères la satisfaction de participer activement au processus décisionnel concernant leur enfant, et leur permettrait de développer un sentiment de confiance envers le personnel de l'école, les soulageant ainsi du poids de devoir revendiquer constamment les services nécessaires à leur enfant. Ces résultats corroborent également ceux de Stoner, Angell, House et Bock (2007) qui concluent que les mères qui se sentent constamment dans l'obligation d'adopter une posture " revendicatrice " et de mener des "batailles " pour leur enfant ne sont pas satisfaites de leur expérience d'intégration. Malheureusement, plusieurs des mères que nous avons rencontrées ont vécu de telles expériences, certaines ayant même été exclues, contre leur gré, des processus décisionnels concernant leur enfant, notamment en ce qui a trait à l'identification des besoins et des objectifs dans le cadre du plan d'intervention. Le gouvernement québécois, dans un document récent, constate qu'un « vrai » partenariat entre l'école et les parents ne constitue pas la norme, actuellement, dans le milieu de l'éducation (MELS, 2010). Dans ce contexte, il n'est donc pas étonnant de constater que le rôle central susceptible d'être joué par les mères n'est souvent pas reconnu par le personnel scolaire, et que ce manque de reconnaissance constitue une source d'insatisfaction et de frustration pour les mères que nous avons rencontrées.

Comme d'autres études l'ont démontré, notre analyse rapporte également que le style de gestion adopté par la direction influence la perception des mères à l'égard de l'intégration scolaire de leur enfant. En effet, une direction qui privilégie une lecture rigide des règlements plutôt que la souplesse et la communication, ne favorise pas le développement de relations positives entre les mères et le personnel, ce qui nuit, d'après les mères, à l'intégration de leur enfant (Gibb, Tunbridge, Chua et Frederickson, 2007; Horrocks, White et Roberts, 2008; lalongo, 2007; Segall et Campbell, 2014; Symes et Humphrey, 2011).

L'analyse du propos des mères souligne qu'en plus d'avoir besoin de soutien et de continuité dans les services (Sapin, Thommen et Wiesendanger, 2007), les mères ont également besoin que le personnel scolaire fasse preuve de compréhension et d'empathie face à leur situation. Cette attitude empathique peut se traduire, par exemple, par le fait de démontrer de l'écoute et de l'intérêt pour les difficultés rencontrées ou par le fait de tenir des propos qui permettent aux mères d'avoir l'impression que ce qui leur arrive est compréhensible et n'est pas lié à 


\section{8}

un manque de compétence parentale. Une telle attitude constitue un élément clé du développement d'un sentiment de confiance, de la part des mères, envers le personnel de l'école. Comme l'a démontré Sabourin (2012) dans son étude portant sur la perception des mères concernant leurs relations avec les intervenantes qui offrent de l'intervention comportementale intensive $(\mathrm{ICl})$ à leur enfant ayant un TSA, il semble que les mères valorisent tout particulièrement le fait de développer un lien de confiance, empreint de respect et d'empathie, avec les professionnelles qui travaillent auprès de leur enfant.

Comme l'ont démontré d'autres études, le manque de compréhension, de la part du personnel, des besoins particuliers des enfants présentant un TSA, amène souvent les intervenantes, non seulement à manquer de sensibilité à l'égard des comportements des enfants (Emam et Farrell, 2009; Whitaker, 2007), mais également à des difficultés pour adapter l'environnement de classe ou les stratégies éducatives à ces enfants (Cappe, 2012; Helps, Newsom-Davis et Callias, 1999; Renty et Roeyers, 2006; Siklos et Kerns, 2006; Unianu, 2012). Le propos des mères que nous avons rencontrées montre qu'elles sont conscientes de l'importance d'outiller le personnel scolaire pour qu'il soit en mesure de comprendre les besoins des enfants présentant un TSA. Les mères déplorent également que le caractère « invisible » du handicap de leur enfant ait pour effet de minimiser les difficultés de l'enfant et nuit à la mise en place des mesures d'adaptation nécessaire à son fonctionnement. Cette situation, comme le décrit Cappe (2012), a un effet sur la relation que les mères entretiennent avec le personnel, car ces dernières souffrent de leur manque de considération concernant les besoins de leur enfant.

L'analyse du propos des mères met en évidence un manque important de formation spécifique au TSA de la part des enseignantes et des accompagnatrices, ce qui est largement corroboré dans la littérature actuelle (Eldar, Thamor et WolfZukerman, 2010; lalongo, 2007; Symes et Humphrey, 2011). Par contre, notre recherche met en lumière le fait qu'au Québec, il arrive que ce soit les mères qui compensent pour ce manque de connaissance à propos du TSA de la part du personnel scolaire en leur transférant leurs connaissances sur les techniques d'intervention ou simplement sur le TSA. Certaines mères adoptent même un rôle plus actif en se déplaçant à l'école pour «former » le personnel. Cette situation peut sembler inquiétante parce qu'elle indique que l'école n'a pas les ressources nécessaires pour former son personnel adéquatement. Même s'il est important, pour ces mères, que leur expertise soit reconnue et même si plusieurs d'entre elles sont prêtes à fournir ce type d'aide pour leur enfant, il n'en demeure pas moins qu'elles considèrent également que l'école a un rôle à jouer dans la formation de son personnel. Il est donc possible que les pratiques, concernant le soutien et la formation des enseignantes et des accompagnatrices, présentent d'importantes variations selon les écoles. En effet, plusieurs mères dénoncent le fait que ces membres du personnel manquent de soutien, ce qui se traduit, entre autres, par un manque d'accès aux professionnels et une faible prise en compte de l'effet, sur la charge de travail, d'intégrer un enfant aux besoins particuliers (par ex., allouer du temps pour préparer le matériel, etc.). On peut donc soulever l'hypothèse selon laquelle le personnel administratif n'est pas suffisamment sensible aux impacts d'une intégration d'un enfant ayant un TSA dans une classe, ou que cette sensibilité ne se traduit pas par la mise en place de ressources nécessaires pour favoriser 
l'intégration. Pourtant, les recherches sont unanimes quant à l'importance du rôle de la direction vis-à-vis la formation du personnel (Eldar, Thamor et WolfZukerman, 2010; lalongo, 2007; Symes et Humphrey, 2011) et de son rôle, selon les enseignantes, d'offrir une conciliation de temps pour adapter le matériel à l'enfant et de l'accès à des ressources professionnelles (Ruel, 2014). D'ailleurs, il semble évident que l'ensemble du personnel scolaire pourrait bénéficier d'une formation spécialisée, surtout dans le contexte où un nombre élevé d'élèves présentant un TSA est scolarisé en milieu ordinaire grâce à la nouvelle philosophie inclusive selon laquelle tout le monde devrait être en mesure d'intervenir auprès de l'enfant afin d'éviter la stigmatisation.

Outre l'investissement supplémentaire que les mères estiment qu'elles doivent faire pour la formation du personnel, ce manque de formation spécifique au TSA mène à une mauvaise interprétation des comportements (Emam et Farrell, 2009) ou l'utilisation d'un matériel peu adapté aux besoins de l'enfant (Helps, Newsom-Davis et Callias, 1999; Segall et Campbell, 2014; Siklos et Kerns, 2006; Unianu, 2012). Nos résultats montrent également que l'incapacité à comprendre les symptômes attribuables au TSA affecte la qualité des plans d'intervention, la qualité des interventions et les choix administratifs faits pour l'enfant (par ex. le choix de l'enseignante, transfert de classe en cours d'année, etc.).

Nous l'avons vu, plusieurs sources d'insatisfaction des mères sont reliées aux comportements, aux attitudes, aux compétences et aux connaissances du personnel scolaire à propos du TSA. Outre le manque de service dénoncé par le Protecteur du Citoyen (2009), la qualité des services offerts pourrait être améliorée. Les résultats de notre recherche semblent indiquer qu'actuellement au Québec, plusieurs des personnes qui travaillent auprès des enfants ayant un TSA pourraient avoir des difficultés à s'adapter à la nouvelle réalité de la pratique inclusive. Par ailleurs, ce constat concernant le manque de formation ce retrouve dans l'ensemble des recherches qui ont porté sur cette question pas uniquement dans la province de Québec (Helps, Newsom-Davis et Callias, 1999; Segall et Campbell, 2014; Siklos et Kerns, 2006). Quelques hypothèses peuvent être évoquées pour expliquer ces difficultés présentent dans le système d'éducation québécois. Tout d'abord, au cours des dernières années, le nombre d'enfants présentant un TSA intégrés en classe ordinaire a connu une forte augmentation (Noiseux, 2012). Cette situation, combinée avec les nouvelles philosophies inclusives adoptées par le gouvernement, explique probablement que le personnel scolaire n'ait pas encore eu le temps de s'adapter à cette nouvelle réalité. Ensuite, comme les enfants présentant un TSA ont des profils très variés, tant sur le plan académique que social (Schwartz, Sandall, McBride et Boulware, 2004; Simpson, Boer-Ott et Smith-Myles, 2003), cela fait en sorte qu'il est nécessaire, pour le personnel, de connaître et maîtriser une multitude de techniques d'intervention. Même si le personnel recevait la formation nécessaire pour comprendre les particularités du TSA, il peut être difficile, pour celui-ci, d'acquérir rapidement l'assurance et la flexibilité que les personnes possédant beaucoup d'expérience auprès de ces enfants réussissent à développer. Cette hypothèse met en évidence l'importance de former tout le personnel scolaire (direction, enseignante, professionnelle et accompagnatrice) le plus tôt possible dans leur cheminent professionnel c'est-à-dire directement dans leur curriculum scolaire comme le suggèrent plusieurs enseignantes (Ghergut, 2010). 
Les résultats de notre recherche concernant l'attitude du personnel scolaire suscitent une toute autre forme de questionnement. Même si l'importance de ce savoir-être pourrait être davantage mise de l'avant dans le cadre de cours et de formations, il demeure surprenant de constater que plusieurs membres du personnel ne semblent pas réaliser d'emblée l'importance de démontrer une ouverture aux enfants présentant des défis particuliers. Pourtant, n'est-il pas évident que des attitudes d'ouverture, de respect et de collaboration sont au cœur de la réussite, et ce, pour tous les enfants? (MEQ, 2003) Un important questionnement s'impose donc à l'ensemble de la société : adoptons-nous les comportements et les attitudes prônés dans les lignes de conduite proposées par le gouvernement pour favoriser le développement des élèves présentant des besoins particuliers? À notre avis, même si des formations étaient mises en place pour que le personnel puisse acquérir des connaissances à propos du TSA, si le savoir-être n'y est pas, l'intégration risque d'être vouée à l'échec. II faut souhaiter qu'une vision plus positive du TSA et de l'intégration puisse se développer lorsque ce trouble sera mieux connu et compris dans le milieu de l'éducation (Dybvik, 2004; lovannone, Dunlap, Huber et Kincaid, 2003; McGregor et Campbell, 2001). Car si des mères nous ont dit que des membres du personnel pouvaient avoir une attitude ouverte tout en manquant de connaissances et d'outil pour aider leur enfant, le manque de savoir-être était plus souvent présent chez des personnes connaissant peu, ou mal, le TSA.

Notre étude comporte évidemment un certain nombre de limites. La méthodologie adoptée et la taille de l'échantillon $(n=18)$ ne nous permettent pas de généraliser les résultats à l'ensemble des familles québécoises dans la même situation. Les mères ayant choisi de participer à l'étude pourraient présenter des caractéristiques différentes de celles de l'ensemble des mères dans cette même situation. Par exemple, les mères ayant vécu plus de difficultés lors de l'intégration de leur enfant pourraient être plus enclines à participer à ce type d'étude afin de revendiquer des services et d'exprimer leur désaccord face à l'organisation des services scolaires au Québec. II est également possible que les mères les plus impliquées dans le processus d'intégration aient été plus intéressées à s'exprimer sur ce sujet. II s'agit, malgré ces limites, d'une recherche qui permet de combler certaines lacunes dans les connaissances, en mettant en lumière le regard que posent les mères sur les caractéristiques du personnel scolaire qui, selon elles, sont susceptibles d'influencer l'intégration de leur enfant. Dans un contexte où le succès et l'échec de l'intégration sont souvent décrits comme liés avant tout au profil de l'enfant, force est de constater que les attitudes, les connaissances sur le TSA et les compétences du personnel, qu'il s'agisse de la direction, des enseignantes et des personnes qui accompagnent l'enfant jouent, selon les mères, un rôle de premier plan. Même si d'autres facteurs sont susceptibles d'affecter la perception des mères à l'égard de l'intégration de leur enfant, il n'en demeure pas moins qu'une attention particulière devrait être portée aux attitudes, aux connaissances sur le TSA et aux compétences du personnel scolaire, car il s'agit de facteurs identifiés par les mères comme déterminants pour le déroulement de l'intégration. Un meilleur arrimage entre la perception des mères et celle du milieu scolaire ainsi que la mise en place de mesures de soutien appropriées, pourraient contribuer à faire de l'école québécoise un milieu plus adapté à l'intégration, ce qui affectera de façon positive la vie des mères et de leurs enfants. 


\section{Références}

American Psychiatric Association (2013). Diagnostic and Statistical Manual of Mental Disorders, Fifth Edition (DSM5) (5e éd.). Arlington, Virginie: American Psychiatric Publishing.

Baker-Ericzèn, M. J., Brookman-Frazee, L. et Stahmer, A. (2005). Stress levels and adaptability in parents of toddlers with and without autism spectrum disorder. Research and Practice for Persons with Severe Disabilities, 30(4), 194-204.

Braun, V. et Clarke, V. (2006). Using thematic analysis in psychology. Qualitative Research in Psychology, 3, 77-101.

Brewin, B. J., Renwick, R. et Schormans, A. F. (2008). Parental perspectives of the quality of life in school environments for children with Asperger syndrome. Focus Autism and other Developmental Disabilities, 23(4), 242-252.

Cappe, É. (2012). Effet de l'inclusion sociale et scolaire sur le processus d'ajustement et la qualité de vie des parents d'un enfant présentant un trouble du spectre autistique. Annales médico-psychologiques, 170, 471-475.

Courcy, I. (2014). Maternité en contexte d'autisme: les conditions matérielles et normatives de la vie des mères de jeunes enfants autistes au Québec (Thèse de doctorat inédite). Université du Québec à Montréal.

des Rivières-Pigeon, C. et Courcy, I. (dir.) (2014). Autisme TSA: quelles réalités pour les parents du Québec? Québec : Presses de I'Université du Québec.

Dybvik, A. C. (2004). Autism and the inclusion mandate. Education Next, 4(1), 42-49.

Eldar, E., Talmor, R. et Wolf-Zukerman, T. (2010). Successes and difficulties in the individual inclusion of children with autism spectrum disorders (ASD) in the eyes of their coordinators. International Journal of Inclusive Education, 14(1), 97-114.
Emam, M. M. et Farrell, P. (2009). Tensions experienced by teachers and their views of support for pupils with autism spectrum disorders in mainstream schools. European Journal of Special Needs Education, 24(4), 407-422.

Ghergut, A. (2010). Analysis of inclusive education in Romania: Results from a survey conducted among teachers. Procedia Social and Behavioral Sciences, 5, 711-714.

Giangreco, M. F., Edelman, S. W., Broer, S. M. et Doyle, M. B. (2001). Paraprofesionnal support of students With disabilities: Literature from the past decade. Exceptional Children, 68(1), 45-63.

Gibb, K., Tunbridge, D., Chua, A. et Frederickson, N. (2007). Pathways to inclusion: Moving from special school to mainstream. Educational Psychology in Practice, 23(2), 109-127.

Granger, S. (2011). Les formes d'implication maternelle et paternelle et les facteurs qui y sont associés en contexte d'intervention comportementale intensive (Essai doctoral inédit). Université du Québec à Montréal.

Helps, S., Newsom-Davis, I. C. et Callias, M. (1999). Autism: The teacher's view. Autism, 3(3), 287-298.

Horrocks, J. L., White, G. et Roberts, L. (2008). Principals' attitudes regarding inclusion of children with autism in Pennsylvania public schools. Journal of Autism and Developmental Disorders, 38, 1462-1473.

lalongo, A. G. (2007). Conditions pour réussir l'intégration scolaire. Université de Montréal.

lovannone, R., Dunlap, G., Huber, H. et Kincaid, D. (2003). Effective educational practices for students with autism spectrum disorders. Focus Autism and other Developmental Disabilities, 18, 150-165. 
MacFarlane, K. et Woolsfon, L. M. (2013). Teacher attitudes and behavior toward the inclusion of children with social, emotional and behavioral difficulties in mainstream school: An application of the theory of planned behavior. Teaching and Teacher Education, 29, 46-52.

McGregor, E. et Campbell, E. (2001). The attitudes of teachers in Scotland to the integration of children with autism into mainstream schools. Autism, 5(2), 189207.

Ministère de l'Éducation du Loisir et du Sport (2008). Des conditions pour mieux réussir! Plan d'action pour soutenir la réussite des élèves handicapés ou en difficulté d'adaptation ou d'apprentissage (EHDAA). Québec: Ministère de l'Éducation, du Loisir et du Sport.

Ministère de l'Éducation du Loisir et du Sport (2010). Document d'appui à la réflexion: Rencontre sur l'intégration des élèves handicapés ou en difficulté. Québec: Ministère de l'Éducation, du Loisir et du Sport

Ministère de l'Éducation du Québec (1999). Politique de l'adaptation scolaire: une école adaptée à tous ses élèves. Québec: ministère de l'Éducation du Québec.

Ministère de l'Éducation du Québec (2003). 2 réseaux, 1 objectif : le développement des jeunes. Québec: Ministère de l'Éducation du Québec.

Ministère de la santé et des services sociaux (1996). L'organisation des services aux personnes autistes, à leur famille et à leurs proches. Québec : Ministère de la Santé et des Services Sociaux.

Noiseux, M. (Juin, 2012). Le trouble envahissant du développement (TED) : l'augmentation de la prévalence poursuit son cours-données finales. Longueuil : Agence de la santé et des services sociaux de la Montérégie, Direction de la santé publique.
Paquet, A., Forget, J. et Giroux, N. (2009). Les comportements d'éducateur soutenant l'intégration en classe ordinaire d'élèves ayant un trouble envahissant du développement. Canadian Journal of Education, 32(3), 420-447.

Protecteur du Citoyen (2009). Sondage réalisé auprès de parents d'enfants âgés de 7ans ou moins et présentant un trouble envahissant du développement (TED) aux fins de l'étude du protecteur du citoyen portant sur les services gouvernementaux qui leur sont destinés. Québec : Assemblé Nationale Québec.

Renty, J. et Roeyers, H. (2006). Satisfaction with formal support and education for children with autism spectrum disorder: the voice of the parents. Child: Care, Health and Development, 32(2), 371385.

Rogé, B. (2008). Autisme, comprendre et agir: Santé, éducation, insertion $\left(2^{\mathrm{e}}\right.$ éd.). Paris : Dunod.

Rousseau, N., Dionne, C., Vézina, C. et Drouin, C. (2009). L'intégration scolaire d'enfants ayant une incapacité : perceptions des parents québécois. Revue canadienne de l'éducation, 32(1), 34-59.

Ruel, M.-P. (2014). Les perceptions des enseignants de classe ordinaire quant à l'intégration d'un élève présentant un trouble du spectre de l'autisme (Essai doctoral inédit). Université du Québec à Montréal.

Sabourin, G. (2012). Parents d'enfants ayant un trouble du spectre de l'autisme en contexte d'intervention comportementale intensive : perception et déterminants de leur détresse (Thèse de doctorat inédite). Université du Québec à Montréal.

Sapin, V. Z., Thommen, E. et Wiesendanger, S. (2007). Les transitions dans les trajectoires des personnes atteintes d'autisme et du syndrome d'Asperger: Le point de vue des familles. Communication présentée Congrès suisse de pédagogie spécialisée Berne 
Schwartz, I. S., Sandall, S. R., McBride, B. J. et Boulware, G. L. (2004). Project DATA (Developmentally Appropriate Treatment for Autism): An inclusive school-based approach to educating young children with autism. Topics in Early Childhood Special Education, 24, 156-168.

Segall, M. J. et Campbell, J. M. (2012). Factors relating to education professionals' classroom practices for the inclusion of students with autism spectrum disorders. Research in Autism Spectrum Disorders, 6, 1156-1167.

Segall, M. J. et Campbell, J. M. (2014). Factors influencing the educational placement of students with autism spectrum disorders. Research in Autism Spectrum Disorders, 8, 31- 43.

Siklos, S. et Kerns, K. A. (2006). Assessing need for social support in parents of children with autism and Down syndrome. Journal of Autism and Developmental Disorders, 36, 921-933.

Simpson, R. L., Boer-Ott, S. R. et SmithMyles, B. (2003). Inclusion of learners with autism spectrum disorders in general education settings. Topics in Language Disorders, 32(2), 116-133.

Starr, E. M. et Foy, J. B. (2012). In Parents' Voices: The education of children with autism spectrum disorders. Remedial and Special Education, 33(4), 207-216.

Starr, E. M., Foy, J. B., Cramer, K. M. et Singh, H. (2006). How are schools doing? Parental perceptions of children with autism spectrum disorders, Down syndrome and learning disabilities: A comparative analysis. Education and Training in Developmental Disabilities, 41(4), 315-332.
Stoner, J. B., Angell, M. E., House, J. J. et Bock, S. J. (2007). Transitions: Perspectives from parents of young children with autism spectrum disorders (ASD). Journal of Developmental and Physical Disabilities, 19, 23-39.

Stoner, J. B., Bock, S. J., Thompson, J. R., Angell, M. E., Heyl, B. S. et Crowley, E. P. (2005). Welcome to our world: Parent perceptions of interactions between parents of young children with ASD and education professionnals. Focus Autism and other Developmental Disabilities, 20(1), 39-51.

Symes, W. et Humphrey, N. (2011). School factors that facilitate or hinder the ability of teaching assistants to effectively support pupils with autism spectrum disorders (ASDs) in mainstream secondary schools. Journal of Research in Special Educational Needs, 11(3), 153-161.

Unianu, E. M. (2012). Teachers' attitudes towards inclusive education. Procedia Social and Behavioral Sciences, 33, 900-904.

Whitaker, P. (2007). Provision for youngsters with autistic spectrum disorders in mainstream schools: what parents sayand what parents want. British Journal of Special Education, 34(1), 170-178. 